\title{
MORPHOMETRIC STUDIES OF CHLOROQUINE INDUCED AUTOPHAGY IN RAT LIVER*
}

\author{
Ann Marie Wisner-Gebhart, Roberta K. Brabec and Robert H. Gray \\ Department of Environmental and Industrial Health, Environmental Cellular Chemistry Laboratory, \\ The University of Michigan, Ann Arbor, MI 48109, U.S.A.
}

Stereological principles were applied to the morphometric analysis of the autophagic process induced by the drug chloroquine (Abraham et al., 1968; Gray et al., 1973). Cellular autophagy is an important event to study because the series of complex subcellular events associated with it appear to help the cell to accommodate to stress. It was hoped that any subcellular changes that did occur during this process could be quantitated through morphometric analysis. Eight control animals were sacrificed, as well as two animals each at 1,2 and $6 \mathrm{hr}$ following chloroquine administration. Five liver samples from each animal were prepared and examined in the TEM. Morphometric methods were used

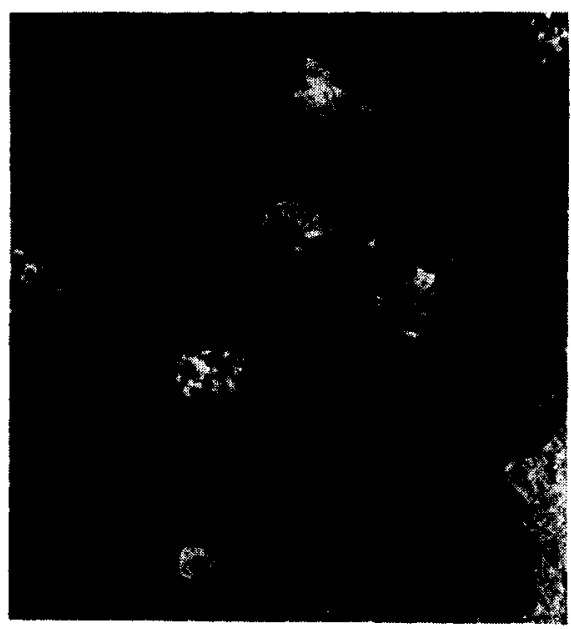

Fig. 1. Electron micrograph of rat liver $2 \mathrm{hr}$ following drug administration. Several autophagic vacuoles (AV) and mitochondria $(\mathrm{M})$ are shown. $\times 5000$.

* Supported by the Michigan Memorial Phoenix Project grant No. 478. to characterize autophagic vacuoles, mitochondria, lysosomes, and peroxisomes according to their numerical density and relative volume within the hepatocyte (Weibel et al., 1969). Figure 1 is an example of a hepatocyte with numerous autophagic vacuoles containing lamellar membranes and/or ingested cytoplasmic organelles. More than 500 times the number of autophagic vacuoles observed in the control animals were found in those treated with chloroquine (Fig. 2). The volume per autophagic vacuole peaks at $2 \mathrm{hr}$ following drug treatment. Figures 3 and 4 illustrate the number per cell and the volume per organelle of the mitochondria, lysosomes and peroxisomes, expressed as a ratio with control values. In Fig. 3, the mitochondria demonstrate fluctuation in their number per cell over time, but they show a definite increase in volume (Fig. 4). Variation in the number of lysosomes per cell can also be seen in Fig. 3, although there is a definite decrease in their

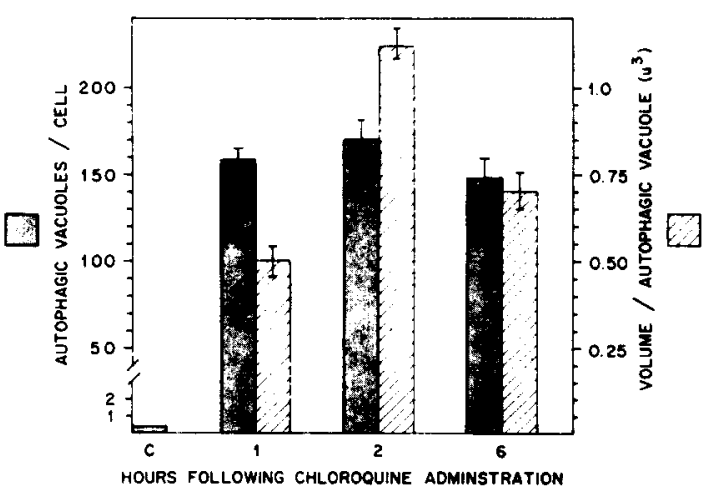

Fig. 2. $\mathrm{C}=$ Control. There is no graph for the volume per autophagic vacuole for the control, because there were too few vacuoles observed in control animals to make a valid comparison. 

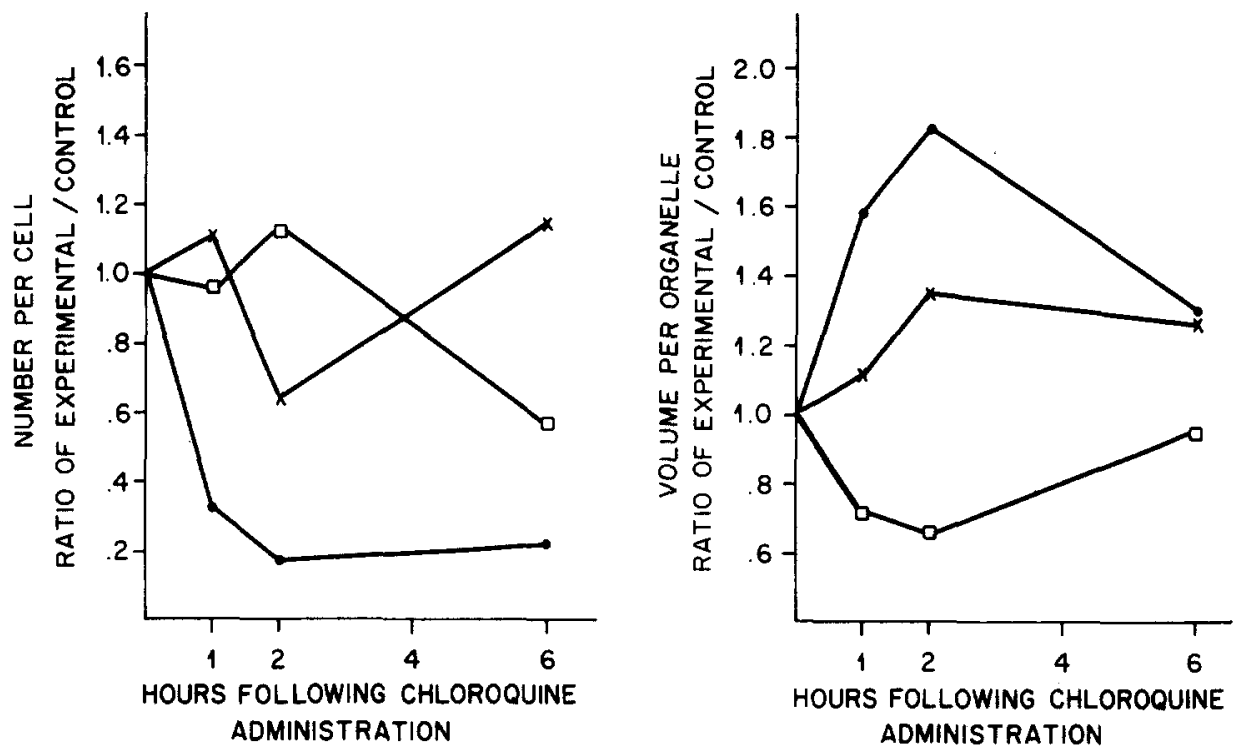

Figs. 3. and 4. Peroxisomes ( $-\square)$; lysosomes $(\square-\square)$; mitochondria $(\times-\times)$.

volume per organelle in Fig. 4. The peroxisomes display a decrease in number while showing an increase in volume. It can be concluded that the frequency of autophagic vacuoles in hepatic tissue can be significantly and rapidly induced following the administration of the drug chloroquine. The other organelles studied also showed distinct quantitative changes. The use of stereo- logical techniques allowed that which had been qualitatively observed to be proven in a quantitative fashion.

\section{REFERENCES}

Abraham, R. et al., 1968. Exp. molec. Path., 12: 148-159.

Gray, R. H. et al., 1973. J. Cell Biol., 59:121a.

Weibel, E. R. et al., 1969. J. Cell Biol., 42: 68. 